\title{
Power Control for Mc-Cdma System with Predicted Csi Using Water Filling Algorithm
}

\author{
Suresh $^{1}$, Mahadevan ${ }^{2}$ \\ ${ }^{I}$ (ECE, Pondicherry Engineering college, India) \\ ${ }^{2}$ (ECE, Pondicherry Engineering College, India)
}

\begin{abstract}
Multi-carrier CDMA technology is a very attractive multiple access technique for next generation wireless communication systems. It combines the advantages of both Orthogonal Frequency Division Multiplexing (OFDM) and Code Division Multiple Access (CDMA) for high data rate transmission. However, interference noise is considered as a major challenge for MC-CDMA systems and significantly affects its performance. The transmission strategy to avoid the interference noise is by its power allocation to each user. The Game theory is an efficient way for examining power control problem in the MC-CDMA technology. The water filling algorithm in game theory perspective is used to allocate proper power for every sub-channel based on channel state information (CSI) with global power constraint in order to improve the capacity of the MCCDMA system. The water filling provides optimal solution if the channel state information is perfect. In realistic high mobility environment, where the Doppler frequency is high, channel state information received at the transmitter is outdated due to CSI feedback delay which results in imperfectness in the channel state information. The capacity achieved using water filling game theory is analysed with Perfect CSI, Imperfect CSI and Predicted CSI. The water filling game theory with Predicted CSI shows improvement in the capacity compared to water filling game theory with Imperfect CSI.
\end{abstract}

Keywords: Capacity improvement, multi-channel wireless communication, power control, water-filling

\section{Introduction}

The Wireless communication is considered to be the fastest growing segment of the communication field. Cellular systems have experienced faster growth over the last decade and as a result there are currently two billion users around the world. Indeed, cellular phones have become a most useful business tool and part of everyday life in most developed countries, and are icreasingly supplanting antiquated wire line communication systems in many developing countries. Due to the advantages of wireless communications, various leading telephone service providers in the world introduced radio telephone. The increasing number of subscribers and the need for the delivery of new services with high quality induced the emergence of successive cellular systems that can be classified in to various generations. Recently, wideband CDMA systems have been developed as a $3 \mathrm{G}$ contender to increase the data rates for the user . However, the large frequency bandwidth of such highspeed links makes them susceptible to inter-symbol interference (ISI). The CDMA and OFDM are independently are not in a position to meet this requirements. Multi carrier code divisible multiple access (MCCDMA) scheme has the capability of simultaneously exploiting the advantages of CDMA and OFDM, a multicarrier modulation scheme.MC-CDMA system is considered to be one of the potential candidates considered potential candidates to fulfil the requirements of next generation (4G) high-speed wireless multimedia communications systems. MC-CDMA systems transmit data over several orthogonal subcarriers. The capacity of MC-CDMA cellular system is mainly limited by the mutual interference generated by the mobile stations and the fading radio channel. A reduction in interference will obviously increase the system capacity. Interference is caused predominantly by the effects of co-channel interference and fast fading channel. These effects can be effectively compensated through perfect power control so that all the subcarrier signals from the transmitter arrive at the same power level and in turn serve to maximize the system capacity.

Though the same amount of power is transmitted through each subcarrier, the received power level at the receiver may have different values. This may lead to significant performance degradation, in terms of BER and reduction in system capacity. In order to alleviate the variations of received power level on different subcarriers power control was introduced. The subchannel power controlled MC-CDMA systems can transmit data with variable power according to the received SINR for each subchannel at the receiver. The efficient way to perform this power allocation is water filling algorithm to allocate power for each subcarrier based on the channel state information from the receiver to the transmitter to improve the capacity of the MC-CDMA system.

\section{Literature Survey}

K. S. Gilhousen, et.al [1], have exhaustively studied the capacity of cellular CDMA system and suggested methods to improve it. These techniques have targeted either the transmitter-side or the receiver-side. 
Transmitter side techniques aim to reduce or avoid the interference that the users experience. Power control is an effective interference management method at the transmitter side of the communication. Receiver side techniques, aim to suppress the interference using advanced receivers. P Patel, et.al [2], discussed that the power control is a useful tool to increase the capacity of CDMA systems. S.P.W Jarot, et.al [3], discussed that a significant amount of work has been reported on transmission power control techniques for single carrier systems, only a few attempts have been made for multi carrier systems like MC-CDMA so far. Though the need for power control in MC-CDMA systems has been highlighted, a practical transmission power control techniques have not been developed. As different subcarriers experience different fades and transmit different numbers of bits, the transmission power levels must be changed accordingly. Daniel Pérez Palomar, et.al [4], discussed the water filling solves the problem of maximizing the capacity by allocating power for sub channels with a global power constraint at the transmitter.Farhad Meshkati, et.al [5], proposed the power control as a non co-operative game in which users choose their transmit powers in order to maximize their utility. They proposed water filling as a non cooperative game for power control and it has the visual interpretation of pouring water over a surface given by the inverse of the subchannel gains to achieve the capacity.

Hanna Bogucka [6], and Chunhui Liu, et.al [7], discussed that based on the channel state information feedback from the receiver to the transmitter and the given power constraint, the Water level is calculated and the appropriate transmission power levels are assigned to subcarriers to maximize the capacity. The water filling (WF) algorithm requires perfect CSI at the transmitter to provide optimal solution. In realistic scenario only imperfect CSI is available. However, the game theoretic model of water filling algorithm will provide good performance with Imperfect CSI Amod J. G. Anandkumar, et.al [8], discussed that in practical wireless communication systems, the transmitters able to receive channel state information (CSI) as feedback from receiver. For a very slowly varying channel, the CSI received may be sufficient for power control. For a fastly varying channel, the CSI received is outdated for power control. However, water filling game theory shows better performance with Imperfect CSI. Alexandra Duel Hallen, et.al [9], discussed that it is necessary to use an efficient power control with a fading prediction algorithm to improve the capacity of the systems in a high mobility environment. They analyzed sinusoidal modelling based prediction and autoregressive modelling based prediction algorithm. They reported that the AR model-based prediction can outperform the deterministic sinusoidal modelling based methods in many fading scenarios. Similarly it is shown in that the AR-based linear predictor outperforms the SoS on real measurement data. Darina Jarinová [10], proposed autoregressive model based CSI prediction to enable an efficient power control, in which the channel state has to be predicted several symbols ahead to compensate for delay in the feedback loop in a fastly changing environment.

The objective of the present work is to implement efficient power control method to improve the capacity of the MC-CDMA system. Water filling rule in game theoretic perspective is considered as an efficient tool used to allocate proper power for every user based on channel state information in order to improve system capacity performance with global power constraint. In high mobility environment, to meet the challenges of Imperfect CSI, Autoregressive model based CSI prediction is used along with water filling game theory in order to improve the capacity of the MC-CDMA

\section{Power Control Using Water Filling Game Theory}

MC-CDMA systems transmit data over several orthogonal subcarriers. The capacity of MC-CDMA cellular system is mainly limited by the mutual interference generated by the mobile stations and the fading radio channel. A reduction in interference will obviously increase the system capacity. Interference is caused predominantly by the effects of co-channel interference and fast fading channel. These effects can be effectively compensated through perfect power control so that all the subcarrier signals from the transmitter arrive at the same power level and in turn serve to maximize the system capacity

\subsection{Game Theory}

Game theory can be viewed as a branch of applied mathematics as well as of applied sciences. It has been used in the social sciences, most notably in economics, but has also penetrated into a variety of other disciplines such as in the field of biology, computer science, philosophy, political science and, recently, wireless and communication networks.

\subsection{Water filling game theory}

A game theoretic perspective for power control in multicarrier system is water filling game theory. The water-filling rule in game theoretic perspective is an efficient tool used to allocate proper power for every user in order to improve system capacity performance with global power constraint. This capacity achieving solution has the visual interpretation of pouring water over a surface given by the inverse of the sub channel gains, hence the name water filling or water pouring. This algorithm defines the way to distribute the power among the frequency channels. The main idea behind is to increase the capacity accumulating the power in the less 
interfered channels. The capacity enhancement is reached by distributing the energy in a water filling manner. Fig.1 represents an example of how the algorithm works and shows the reason for its name: thinking of the noise and interference as the bottom of a vessel, the algorithm fills it with power as if it was a sort of liquid, leaving a flat surface on its top. The unshaded portion of the graph represents the inverse of the power gain of a specific channel. The portion representing the shadow represents the power allocated or the water

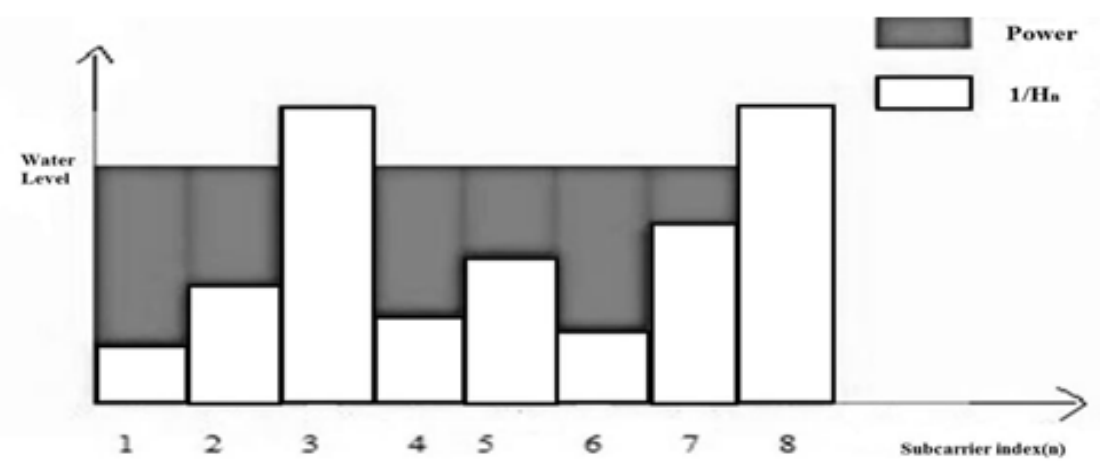

Fig 1. Water filling algorithm

The water filling algorithm is explained in the following steps:

1.Initially take the sum of the Total Power $P_{t}$ and the Inverse of the channel gain to noise ratio.It gives the complete area in the waterfilling and inverse power gain.

$$
\left(P_{t}+\sum_{n=1}^{N} \frac{1}{H_{n}}\right) \text { for } \mathrm{n}=1,2, \ldots \ldots \mathrm{N}
$$

where,

$$
\begin{aligned}
& \mathrm{P}_{\mathrm{t}} \text { is Total Power Available } \\
& \frac{1}{H_{n}} \text { is inverse of channel gain }
\end{aligned}
$$

$\mathrm{n}$ is subcarrier index

2.Calculate the water level by considering the total power constraint and the inverse of the channel gain to noise ratio.

$$
\frac{\left(P_{t}+\sum_{n=1}^{N} \frac{1}{H_{n}}\right)}{\sum \text { Channels }} \quad \text { fon }=1,2, \ldots . . \mathrm{N}
$$

3. The power values of each subchannel are calculated by subtracting the inverse of channel gain to noise ratio of each subchannel to the water level.

$$
\frac{\left(P_{t}+\sum_{n=1}^{N} \frac{1}{H_{n}}\right)}{\sum \text { Channels }}-\frac{1}{H_{n}} \quad ; \text { for } \mathrm{n}=1,2, \ldots . . \mathrm{N}
$$

4. The capacity achieved is given by

$$
\log _{2}\left(1+P_{n^{*}} H_{n}\right)
$$

This algorithm provides the maximum capacity by allocating more power to the frequency bands with higher channel gain to noise ratio. 


\subsection{System Model}

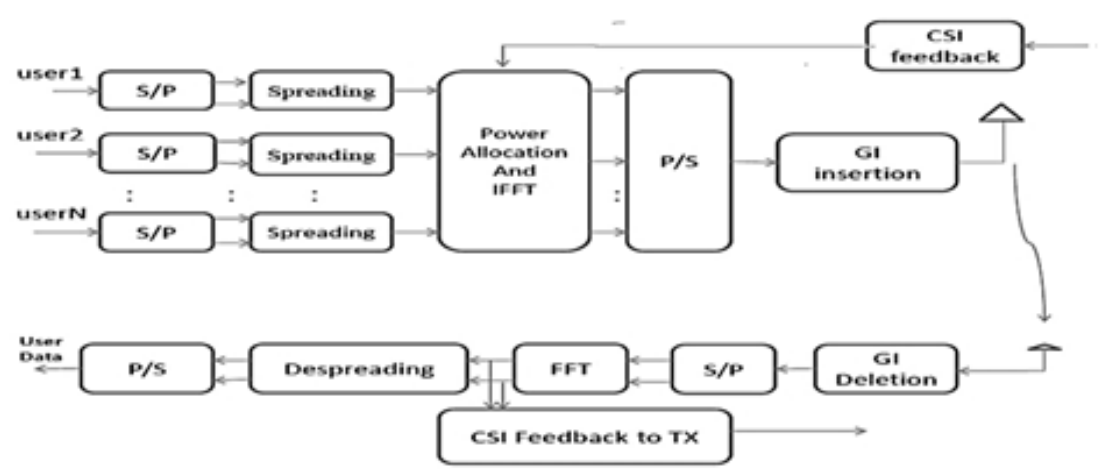

Fig.2 System Model

The power control scheme for MC-CDMA system using water filling game theory is depicted in Fig 2. In MC-CDMA system each user spreads the original data stream over each subcarrier by using walsh hadamard code. The spreaded data is modulated onto the subcarrier with the help of IFFT operation. The transmitter allocates power for each subcarrier using water filling game theory based on the channel state information received as feedback from the receiver. The symbol ' $U$ ' in the base station ' $b$ ' of an MC-CDMA system is given by equation (5).

$$
Y^{u}=H^{u} S^{u} \sqrt{P^{u}} X^{u}
$$

where,

$$
\begin{aligned}
& \mathrm{Y}^{\mathrm{u}} \text { is the MC-CDMA symbol vector. } \\
& \mathrm{H}^{\mathrm{u}} \text { is each user's channel fading. } \\
& \mathrm{S}^{\mathrm{u}} \text { is code matrix } \\
& \mathrm{P}^{\mathrm{u}} \text { is each user's transmit power. } \\
& \mathrm{X}^{u} \text { is the active user's symbol. }
\end{aligned}
$$

The water filling algorithm allocates power to the subcarrier for each user based on the channel state information. The receiver estimates the channel and provides the feedback to the transmitter for power control using water filling game theory.

\section{Predicted Csi}

In most mobile radio systems, the CSI is estimated at the receiver and fed back to the transmitter. The perfect feedback of CSI has been an essential assumption for most resource allocation schemes. In realistic high mobility environment, perfect channel state information is not always available since the wireless communication channel is much more volatile than its wired counterpart. However, the water filling algorithm provides optimal solution through power allocation to the subcarriers for achieving the capacity of the MCCDMA system only if the CSI is actual and valid. In realistic environment, there is always a time difference between the time that the channel is measured and the time that the corresponding transmission is made which results in the imperfectness in the channel state information. The impact of imperfect CSI would degrade the performance achieved through power control using water filling game theory because the power allocation to the subcarriers using water filling game theory that strongly relies on the perfect channel state information. Therefore, to enable efficient power control using water filling game theory in order to improve the capacity of the system, prediction is required to maintain the reliability of the channel state information at the transmitter.

\subsection{CSI Prediction}

In open-loop channels, current CSI is not sufficient since future channel conditions need to be known to adapt transmission parameters. To realize the potential of adaptive transmission methods, theses channel variations have to be predicted at least several milliseconds, or tens to hundreds of data symbols, ahead for efficient transmission. 


\subsection{Autoregressive model based CSI prediction}

The objective of the Autoregressive model based long range prediction is to forecast the future values of the channel state information far ahead to enable efficient transmission in high mobility environment. In AR model based linear prediction, the fading channel gains $g$ are assumed to be generated as an autoregressive process. Then the prediction of the future CSI sample $g(n)$ can be approximated as a linear combination of $M$ previous samples and is given by

$$
g(n)=\sum_{j=1}^{M} a_{j} \cdot g(n-i)
$$

where $\mathrm{M}$ is the order of the AR process, $\mathrm{n}$ is time index, and $\mathrm{a}_{\mathrm{i}}$ are AR coefficients.AR coefficients are obtained by solving the below equation written in matrix form

$$
a=R^{-1} r
$$

where $a=\left[a_{1}, a_{2} \ldots \ldots \ldots . . . a_{M}\right]^{T}, R$ is a autocorrelation matrix $(M x M)$ with coefficients $R_{i j}=E[g(n-i) g(n-j)]$ and $r$ is the corresponding autocorrelation vector $(M x 1)$ with the coefficients $r_{j}=E[g(n) g(n-j)]$. Since channel statistics usually change over time the AR coefficients are regularly adapted during the prediction process and can be estimated from the observation samples.

If Channel state information is required several steps ahead, the prediction using (6) is repeated a given number of times in order to enable efficient power control using water filling game theory. This predicted CSI is applied for power control using water filling game theory in high mobility environment which improves the capacity of the MC-CDMA system.

\subsection{Simulation results for perfect CSI}

\section{Simulation Results}

The Capacity and Bit Error Rate performance of the MC-CDMA System achieved through power control with perfect Channel State Information (CSI) is analysed through simulation using Matlab. The simulation parameters are shown in Table 4.1. The simulation results for capacity achieved through power control using water filling algorithm with perfect CSI, imperfect CSI and predicted CSI is shown and the discussion about the results achieved is also analyzed.

Table.1 Simulation Parameters For Power Control Using Water Filling Game Theory

\begin{tabular}{|l|l|}
\hline PARAMETERS & SPECIFICATIONS \\
\hline Number of Subcarriers & 256 \\
\hline Spreading code & Walsh Hadamard \\
\hline Spreading factor & 256 \\
\hline Channel Model & Rayleigh fading \\
\hline Doppler frequency $(\mathrm{Hz})$ & 4,150 \\
\hline Modulation type & QAM \\
\hline AR model order & 60 \\
\hline Power available $\mathrm{P}_{\mathrm{t}}(\mathrm{Watt})$ & 1 \\
\hline SINR range(dB) & 0 to 25 \\
\hline
\end{tabular}

Fig. 3 shows the comparison of the capacity achieved by the MC-CDMA system through power control with and without water filling game theory considering perfect channel state information. The channel state information is considered as perfect by assuming the channel as a slowly varying channel and power control has been performed. The simulation result shows there is an improvement in the capacity of the MC-CDMA system achieved through power control with water filling game theory compared to capacity achieved through power control without water filling game theory.

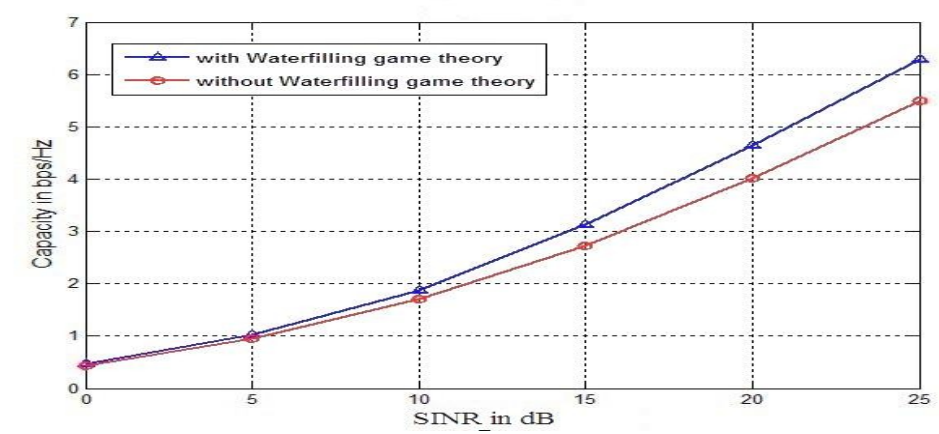

Fig.3 Capacity enhancement through power control using water filling game theory with perfect CSI 
Fig. 4 shows the comparison of the capacity achieved by the MC-CDMA system through power control with and without water filling game theory considering imperfect channel state information. The simulation is done by considering the channel as a fast varying channel with high Doppler frequency which makes the Channel state information as out dated due to delay in the feedback loop. This outdated CSI is called as Imperfect CSI and it has been used for performing power control.

The simulation results shows that the power control with water filling game theory shows good performance in the capacity of the MC-CDMA system compared to capacity achieved through power control without water filling game theory. However, the water filling game theory shows some amount of degradation in the capacity achieved with Imperfect CSI when compared to condition the channel state information is perfect.

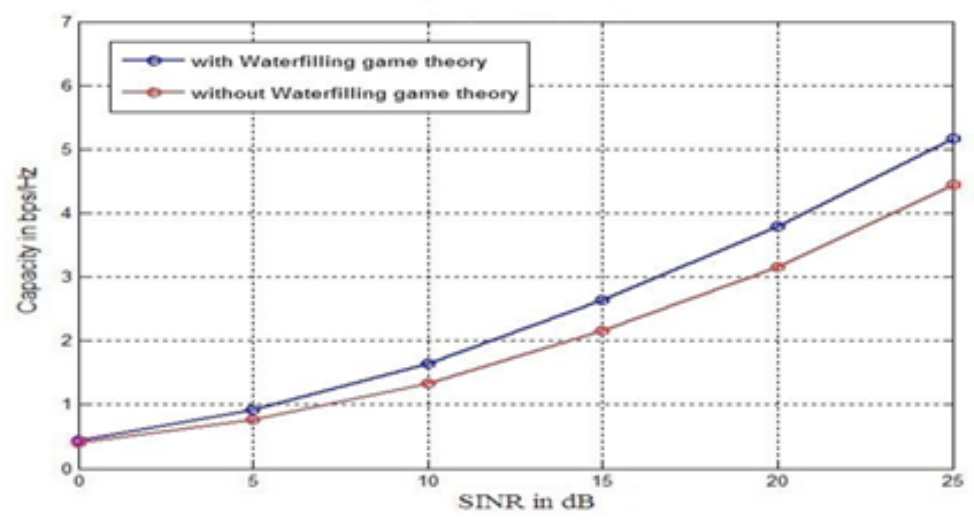

Fig.4 Capacity enhancement through power control using water filling game theory with imperfect CSI

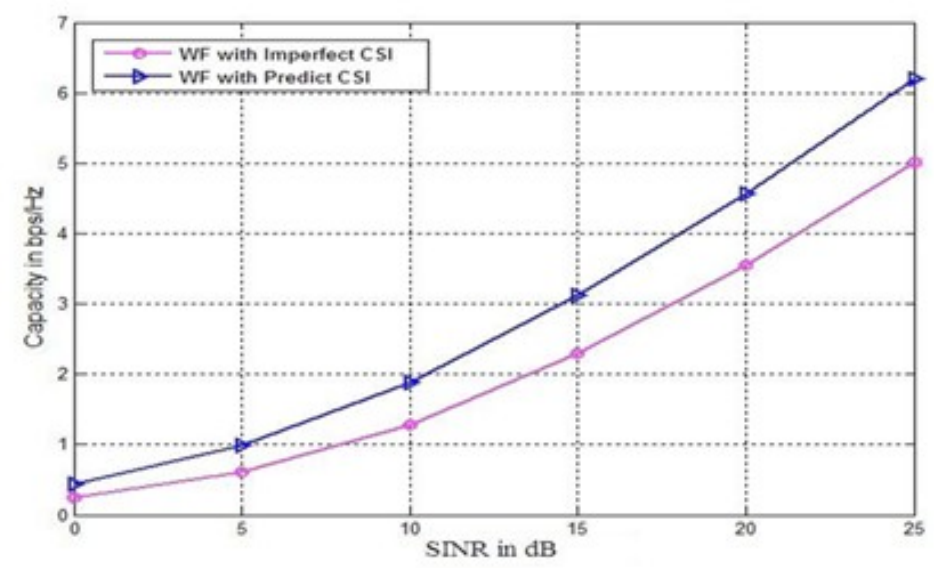

Fig.5 Capacity enhancement through power control using water filling game theory with predicted CSI

Fig. 5 shows the comparison of the capacity achieved by the MC-CDMA system through power control with water filling game theory considering imperfect channel state information and predicted channel state information. The simulation is done by considering the channel as a fast varying channel with high Doppler frequency which makes the Channel state information as out dated due to delay in the feedback loop. To compensate for the delay in the feedback loop, channel state information has to be predicted several symbols ahead in order to overcome the channel mismatch errors.

For this purpose, an autoregressive model based prediction algorithm is used to provide reliable prediction of the channel state information. It continuously tracks the short time history of the channel and approximates a future CSI sample ahead as a linear combination of a current and several recent samples. This predicted CSI is applied for power control using water filling game theory in high mobility environment. The simulation result shows there is an improvement in the capacity of the MC-CDMA system achieved through power control with water filling game theory using predicted CSI when compared to capacity achieved through power control using water filling game theory with imperfect CSI 


\section{Conclusion}

We proposed the power control method for MC-CDMA system using water filling algorithm in game theoretic perspective to allocate proper power for every sub-channel with global power constraint with predicted Channel State information in order to improve the capacity of the MC-CDMA system in realistic high mobility environments. The proposed method shows that the water filling game theory with Predicted Channel State Information shows improvement in the capacity of the MC-CDMA system compared to the capacity achieved using water filling game theory with imperfect Channel State Information in a high mobility environment.

\section{References}

[1]. K.S.Gilhousen, I.M.Jacobs, R.Padovani, A.J. Viterbi, L.A.Weaver Jr., and C.E. Wheatley III, "On the capacity of a cellular CDMA system," IEEE Transactions on Vehicular Technology, vol.40, no.2, pp.303-312, May 1991

[2]. P.Patel and J.Holtzman, "Analysis of a simple successive interference Cancellation scheme in a DS CDMA system," IEEE Journal on selected areas in communication, vol.12, no.5, pp. 796-807, June 1994.

[3]. S.P.W Jarot and M. Nakagawa, "Transmission powercontrol technique for the reverse link of OFDM-DS-CDMA system", Proceedings of IEEE International Symposium on Computers and Communications, Red Sea, Egypt, pp.331-337, July 1999.

[4]. D.P.Palomar and J.R.Fonollosa, "Practical algorithms for a family of waterfilling solutions," IEEE Transaction on signal processing, vol.53, no.2, pp.686 - 695, Feb.2005.

[5]. Farhad Meshkati, Mung Chiang, and H.Vincent Poor, "A Game-Theoretic approach to energy-efficient Power Control in Multicarrier CDMA Systems,” IEEE Journal on selected areas in communications, vol. 24, no. 6, pp.1115 - 1129 ,June 2006.

[6]. Hanna Bogucka, "Game theoretic model for the OFDM water-filling algorithm with imperfect Channel State Information," Proceedings of IEEE International Conference Communications,ICC 08,Beijing,China, pp.3814-3818,May 2008.

[7]. Chunhui Liu, Anke Schmeink and Rudolf Mathar, "Efficient power allocation for OFDM with imperfect Channel State Information," Proceedings of IEEE International Conference Wireless Communications, Networking and Mobile Computing, WiCom 09, Beijing, China ,pp.1-4, September 2009.

[8]. Amod J. G. Anandkumar, Animashree Anandkumar, Sangarapillai Lambotharan, and Jonathon A. Chambers, "Robust rate maximization game under bounded channel uncertainty," IEEE Transactions on Vehicular Technology, vol. 60, no. 9,pp. 44714486, November 2011.

[9]. Alexandra Duel Hallen, Shengquan $\mathrm{Hu}$ and Hans Hallen, "Fading channel prediction for mobile radio adaptive transmission system," Proceedings of IEEE Adaptive modulation and transmission in wireless systems ,San Francisco, California ,vol. 95, pp.2299-2313, December 2007.

[10]. Darina Jarinova, "On Autoregressive model order for long-range prediction of fast fading wireless channel," Springer journal on telecommunication system, vol.52, no.6, pp.1533-1539, July 2011 